\title{
Intestinal absorbability of three Radix Puerariae isoflavones including daidzein, daidzin and puerarin
}

\author{
Li Zhang ${ }^{1}$, Antony Kin Pan Siu', Ge Lin² and Zhong Zuo ${ }^{1 *}$
}

\begin{abstract}
Background: Radix Puerariae (Gegen) contains abundant isoflavones in the forms of glycosides and aglycones, such as daidzein, daidzin and puerarin. This study aims to investigate the intestinal absorbability and mechanism of these three structurally related isoflavones.

Methods: The bi-directional transport of these three isoflavones in Caco-2 monolayer model was performed to evaluate their absorbability and involvement of transporters in Transwell. In vitro incubation of daidzin and puerarin with rat intestinal microvilli preparation was conducted to estimate their potential form of absorption in vivo.

Results: Daidzein demonstrated passive diffusion transport while puerarin did not. Daidzin showed basolateral-toapical transport and the absorption extent could be reduced by 50\% in the presence of MK571, a multidrug resistance-associated protein inhibitor (MRP). The in vitro incubation study of daidzin and puerarin indicated that daidzin was hydrolyzed to daidzein whereas puerarin remained unchanged.

Conclusion: While daidzein was transported more efficiently, puerarin was resistant to intestinal hydrolysis and inefficiently transported across intestinal epithelium. Daidzin demonstrated a low intestinal absorbability due to a significant efflux transport mediated by MRPs. Daidzin was likely to be hydrolyzed by intestinal microvilli and subsequently released daidzein for intestinal absorption.
\end{abstract}

\section{Background}

Radix Puerariae, a Chinese medicinal plant, contains three major isoflavones, namely daidzein, daidzin and puerarin (Figure 1). Daidzin, which was reported to inhibit aldehyde dehydrogenase-2 (ALDH-2) in isolated mitochondria, may be useful as an anti-alcohol agent $[1,2]$. Puerarin is used to relieve thirst and treat common cold and neck stiffness due to hypertension [3].

Despite various pharmacological activities reported for isoflavones, only a few pharmacokinetics studies on the soybean isoflavones were reported [4,5]. Izumi et al. found that the absorption rate of aglycones were higher than their corresponding glycosides due to their higher lipophilicities and smaller molecular sizes [5]. Setchell et $a l$. indicated that isoflavone glycosides such as daidzin and genistin in the blood sample were barely detectable

\footnotetext{
* Correspondence: joanzuo@cuhk.edu.hk

'School of Pharmacy, Faculty of Medicine, The Chinese University of Hong

Kong, Shatin, New Territories, Hong Kong SAR, China

Full list of author information is available at the end of the article
}

whereas a large amount of isoflavone aglycone and its conjugated glucuronides were found in blood [6]. Another study suggested that the in vivo bioavailability of isoflavone glycosides could be enhanced by increasing the activities of beneficial intestinal bacteria which were capable of hydrolyzing the glycosides to their corresponding aglycones [7]. Moreover, isoflavones were reported to undergo enterohepatic recirculation $[8,9]$, suggesting that certain transport mechanisms other than passive diffusion may involve in the absorption of isoflavones such as multi-resistance protein (MRP) transporters $[10,11]$. On the other hand, puerarin may exhibit different pharmacokinetics because the intestinal $\beta$-glucosidase is reported to be weakly effective in catalyzing the hydrolysis of puerarin like C-glucoside [12]. Yasuda et al. [12] showed that puerarin was detected in the blood and that conjugated metabolites of both puerarin and daidzein were found in urine, suggesting that puerarin could be absorbed in its intact form while part of it could be hydrolyzed to daidzein before being absorbed 
<smiles>O=c1c(-c2ccc(O)cc2)coc2cc(OC(O)C(O)C3C(O)CCOCC3O)ccc12</smiles>

B<smiles>O=c1c(-c2ccc(O)cc2)coc2cc(O)ccc12</smiles>

C<smiles>O=c1c(-c2ccc(O)cc2)coc2c(C3OC(O)C(O)C(O)C3O)c(O)ccc12</smiles>

Figure 1 Chemical structures of the three selected isoflavones. A Daidzin; B Daidzein; C Puerarin.

in the gastrointestinal tract. Kim et al. demonstrated the intestinal bacterial mediated biotransformation of puerarin to daidzein [13].

Previous findings focused on the intestinal bacterial hydrolysis during the absorption of isoflavone glycosides. For in vivo studies, Caco-2 cell monolayer model [14] was chosen for the investigation of the absorption mechanisms of the three isoflavones and the comparison of absorption between the glycoside and its aglycone in a controlled environment. This study aims to investigate the intestinal absorption mechanisms of daidzin, daidzein and puerarin by using human Caco-2 cell monolayer model as well as in vitro rat microvillus hydrolysis model.

\section{Methods}

\section{Chemical and reagents}

Daidzin was purchased from Fluka Chemie (Germany). Puerarin and daidzein were provided by DH Chen from the Institute of Medicinal Plant Development, Chinese Academy of Medical Sciences, China. Analytical-graded acetonitrile (ACN) were purchased from Riedel-de Haën (Germany). Sodium dihydrogenphosphate dihydrate was purchased from BDH Chemical (UK). Analytical grade dimethyl sulphoxide (DMSO) was from Lab-Scan Analytical Sciences (Thailand). Phosphate buffered saline tablets were purchased from Sigma Chem Co (USA). MK571 was supplied by Biomol Research Laboratory (USA).

Caco-2 human colon carcinoma cell line was obtained from American Type Culture Collection (ATCC number HTB37, USA). Dulbecco's Modified Eagle's Medium, fetal bovine serum, nonessential amino acid, L-glutamine and trypsin-EDTA used in cell culture were purchased from GibcoBRM (USA). Transwell ${ }^{\circledR}$ inserts (sterile Polycarbonate filter) were purchased from Corning Costar Corporation (USA).

\section{Physicochemical characteristics of the selected isoflavones \\ Stability of the selected isoflavones in transport buffer}

The transport buffer $\left(\mathrm{PBS}^{+}\right)$used in the Caco-2 monolayer model was prepared as previously described [15]. Briefly, $20 \mu \mathrm{g} / \mathrm{ml}$ of puerarin and daidzin and $0.5 \mu \mathrm{g} / \mathrm{ml}$ of daidzein solutions in $\mathrm{PBS}^{+}$at $\mathrm{pH} 6.8$ or $\mathrm{pH} 7.4$ were kept in $37^{\circ} \mathrm{C}$ water bath. For stability tests at $37^{\circ} \mathrm{C}$, samples were taken at $0,15,40,75,120$ and 180 minutes. At each time interval, $300 \mu \mathrm{l}$ of isoflavone solution was taken and then acidified with $50 \mu \mathrm{l}$ of $0.425 \%$ phosphoric acid. All experiments were conducted in triplicates. The amount of selected isoflavones remained in $\mathrm{PBS}^{+}$ was determined with high-performance liquid chromatography (HPLC).

\section{Partition coefficients of the selected isoflavones}

The aqueous solution $(10 \mathrm{ml})$ containing individual isoflavones was mixed with octanol $(3 \mathrm{ml})$. The mixtures were equilibrated in tube rotator for 24 hours followed by sampling from the aqueous layers and determining concentrations of the isoflavones by HPLC. The test was performed in triplicates for each isoflavone. The partition coefficients $(D)$ at both $\mathrm{pH} 7.4$ and $\mathrm{pH} 6.8$ were obtained through the following equation:

$$
\log D=\log \left([\text { isoflavone }]_{\text {octanol phase }} /[\text { isoflavone }]_{\text {aqueous phase }}\right)
$$

Bidirectional transport studies of the selected isoflavones in the Caco-2 monolayer model

\section{Caco- 2 cell culture}

The Caco- 2 cells were cultured and seeded in the Transwell ${ }^{\circledR}$ inserts $\left(24 \mathrm{~mm}, 0.4 \mu \mathrm{m}\right.$ pore size, $4.71 \mathrm{~cm}^{2}$, polycarbonate filter) according to our previous study [15]. Cells used for the current study were from passage 35-40. The transport experiments were carried out at 23-25 days after seeding.

Apical-to-basolateral $(A \rightarrow B)$ transport studies

Upon removal of culture medium, the Transwells ${ }^{\circledR}$ were pre-equilibrated with $\mathrm{PBS}^{+}$at $37^{\circ} \mathrm{C}$ for 15 minutes. Then 
$1.5 \mathrm{ml}$ of the isoflavone solution was loaded to the apical (donor) chamber of the Transwell ${ }^{\circledR}$ while $2.6 \mathrm{ml}$ of blank $\mathrm{PBS}^{+}$was loaded to the basolateral (receiver) chamber. For the transport study of daidzein, $0.5 \mathrm{ml}$ of the sample was taken from the receiver side at certain time interval (15, 30, 45, 60 and 90 minutes) followed by replacing with an equal volume of blank $\mathrm{PBS}^{+}$. Samples were also obtained from the donor side at $90 \mathrm{~min}$ utes. For the transport studies of puerarin and daidzin, samples were taken at 30,60, 90, 120, 150 and $180 \mathrm{~min}$ utes from receiver side and at 180 minutes from the donor side. All Caco-2 transport studies were performed at least in triplicates.

\section{Basolateral-to-apical $(B \rightarrow A)$ transport studies}

Procedure was similar to the $A \rightarrow B$ transport studies except for the donor side and the receiver side was reversed. Loading solution $(2.6 \mathrm{ml})$ was added into the basolateral side and $1.5 \mathrm{ml}$ of the blank $\mathrm{PBS}^{+}$was added to the apical side. Samples were taken from the receiver (apical) side at the same time points and at the end of the experiment from the donor (basolateral) side as mentioned for the $\mathrm{A} \rightarrow \mathrm{B}$ studies. The studies were also performed at least in triplicates.

\section{Basolateral-to-apical transport studies of the selected isoflavones in the presence of MK571}

The study was performed only for isoflavones that had shown a favorable $\mathrm{B} \rightarrow \mathrm{A}$ transport in the bi-directional transport studies. As demonstrated in our previous study [15], MK571 is a MRP inhibitor. To investigate the influence of MRP on the efflux transport of the selected isoflavones, we preloaded $1.5 \mathrm{ml}$ of MK571 (50 $\mu \mathrm{M})$ at apical side and $2.6 \mathrm{ml}$ of MK571 $(50 \mu \mathrm{M})$ at the basolateral side respectively. The $\mathrm{B} \rightarrow \mathrm{A}$ transport experiments of the selected isoflavones in the presence of MK571 $(50 \mu \mathrm{M})$ in the both apical and basolateral chambers were then performed following the method described above. The studies were performed in triplicates.

\section{Potential hydrolysis of daidzein glycosides during intestinal absorptions}

Male Sprague-Dawley rats, supplied by and bred at the Laboratory Animal Service Center at The Chinese University of Hong Kong, were housed in an air-conditioned room under a 12/12-hour light/dark cycle. The experiment was conducted after being approved by the Animal Ethics Committee of The Chinese University of Hong Kong. The rat intestinal microvilli preparations (protein content: $0.43 \mathrm{mg} / \mathrm{ml}$ ) were collected as previously described [16] and incubated with daidzin and puerarin $(50 \mu \mathrm{M})$ at $37^{\circ} \mathrm{C}$ for two hours before we added the same volume of ice-cold methanol to terminate the reaction.

\section{Sample treatment and analysis}

Samples obtained in the Caco-2 transport studies were acidified with $0.425 \%$ phosphoric acid at a sample to acid ratio of 150:25 (v/v). An aliquot of acidified samples $(100 \mu \mathrm{l})$ was injected into the HPLC for the measurement of the isoflavone concentrations. Waters 2695 separation modules and Waters 996 photodiode array detector were used in the HPLC analyses of the Caco-2 samples. A reversed-phase column $(100 \times 4.6 \mathrm{~mm} 5 \mu$ Apex ODS Symm, Jones Chromatography, USA) equipped with a guard column $(7.5 \times 4.6 \mathrm{~mm} 5 \mu$ Spherisorb ${ }^{\circledR}$ ODS-2 $C_{18}$, Waters, USA]) was used for the analyses of all three isoflavones. In order to shorten sample-running time, three separated HPLC methods were used for each isoflavone.

For the analysis of daidzein, an isocratic mobile phase of $27 \% \mathrm{ACN}$ in $0.025 \mathrm{M}$ phosphate buffer ( $\mathrm{pH} 2.5)$ at a flow rate of $1 \mathrm{ml} / \mathrm{min}$ was used for the analysis of daidzein. For the analysis of puerarin, a gradient mobile phase consisting of $\mathrm{ACN}$ and $0.025 \mathrm{M}$ phosphate buffer (pH2.5) was used. The gradient program started with a 2-minute run of $10 \% \mathrm{ACN}$ and $90 \%$ of phosphate buffer, then the percentage of $\mathrm{ACN}$ was increase to $15 \%$ in 10 minutes followed by a decrease of $10 \%$ in the following three minutes and maintaining the percentage for another five minutes. Similar to puerarin, a gradient mobile phase containing ACN and $0.025 \mathrm{M}$ phosphate buffer ( $\mathrm{pH} 2.5$ ) was used for the detection of daidzin. The elution started with 18\% ACN and $82 \%$ buffer, followed by an increase of the proportion of ACN to $30 \%$ in the next two minutes and then maintaining for three minutes before returning to the initial condition in two minutes and remaining with the same elution condition for another four minutes. Daidzein was detected at 300 $\mathrm{nm}$ whereas daidzin and puerarin were detected at 250 $\mathrm{nm}$. The detection limits of the selected isoflavones were the lowest concentration of the compounds that could produce peak height three times greater than that of the background noise. The concentrations of prepared isoflavone standard solutions ranged from 0.0125$1 \mu \mathrm{g} / \mathrm{ml}$.

Samples from the in vitro incubation studies were centrifuged at 16,000 $\times g$ (Eppendorf, Germany) for five minutes; $50 \mu \mathrm{l}$ of the supernatant of the samples were obtained and injected into the HPLC for analysis. In order to monitor daidzin, puerarin and daidzein in a single run for the samples from the hydrolysis study, we modified the above HPLC method for simultaneous detection of the three compounds. The analytes were separated with an HPLC column (BDS reversed phase column, $25 \mathrm{~cm} \times 4.6 \mathrm{~mm}$ id; $5 \mu \mathrm{m}$ particle size, Thermo Hypersil, UK) and eluted by the linear gradient elution at a flow rate of $1 \mathrm{ml} / \mathrm{min}$. The gradient started with 
$95 \%$ of water with $0.01 \%$ aqueous acetic acid (A) and 5\% of ACN containing $0.01 \%$ acetic acid (B), changed linearly to $83 \% \mathrm{~A}$ and $17 \% \mathrm{~B}$ in 30 minutes; $70 \% \mathrm{~A}$ and $30 \%$ $\mathrm{B}$ in $30-40$ minutes; $50 \% \mathrm{~A}$ and $50 \% \mathrm{~B}$ in $40-45$ minutes; and then back to the initial composition in 15 minutes followed by ten minutes after equilibrium.

\section{Data analysis}

The apparent permeability coefficient, $P_{a p p}(\mathrm{~cm} / \mathrm{s})$, was calculated as follows [14].

$$
P_{a p p}=\frac{d C / d T \times V}{\left(A \times C_{o}\right)}
$$

where $d C / d T$ is the initial slope of the plot of cumulative isoflavone concentrations versus time; $V$ is the volume of receiver chamber $(\mathrm{ml}) ; A$ is the apparent surface area of the monolayer; $C_{o}$ is the initial concentration in donor site $(\mu \mathrm{mol} / \mathrm{ml})$. In addition to $P_{a p p}$, the percentage of recovery was calculated for each transport study to determine the extent of absence of isoflavone, which may be due to cell uptake or metabolism of the selected isoflavones after passing through the Caco- 2 cells. It was calculated as follows.

Recovery\% = Final amount (in mole) of isoflavone in donor and receptor side/Initial amount (in mole) of isoflavone in donor side

The reported values are expressed as mean \pm standard deviation (SD). Unpaired Student's $t$-test was used to evaluate the statistically significance of difference between two groups. A value of $P<0.05$ was considered statistically significant for all tests.

\section{Results}

\section{HPLC analysis}

The calibration curves of daidzein, puerarin and daidzin were linear throughout the studied concentration range with correlation coefficients greater than 0.999 . The detection limits of these three isoflavones were $50 \mathrm{ng} / \mathrm{ml}$ for daidzein, $25 \mathrm{ng} / \mathrm{ml}$ for daidzin and $12.5 \mathrm{ng} / \mathrm{ml}$ for puerarin. Inter-day verification was conducted with three sets of calibration curve determined at different days. Intra-day verification was performed on three sets of data measured on the same day. Analyses of all isoflavones showed percentage deviation of less than $10 \%$ for all concentrations tested.

\section{Physicochemical characteristics of the selected isoflavones}

After three hours of incubation at pH6.8/pH7.4, $95.50 \pm$ $0.84 \% / 97.73 \pm 3.43 \%, 105.00 \pm 2.72 \% / 106.74 \pm 4.20 \%$ and $108.66 \pm 2.16 \% / 108.11 \pm 5.21 \%$ of daidzein, puerarin and daidzin remained respectively, indicating that the selected isoflavones were considerably stable in $\mathrm{PBS}^{+}$ under all $\mathrm{pH}$ conditions and suitable for the Caco-2 transport studies. To mimic the physiological $\mathrm{pH}$ of human intestine, we chose pH6.8 for the transport studies.

The $D$ of the isoflavones at $\mathrm{pH} 7.4$ was $0.06 \pm 0.02$ for puerarin, $0.80 \pm 0.03$ for daidzin and at least $2.58 \pm 0.02$ for daidzein which corresponded to the order of their lipophilicities, ie daidzein $>$ daidzin $>$ puerarin. The $D$ at $\mathrm{pH} 6.8$ was also measured which was greater than 2.58 $\pm 0.02,0.34 \pm 0.03,-0.42 \pm 0.15$ for daidzein, daidzin, puerarin respectively. The order of lipophilicities of the studied isoflavones at the two studied $\mathrm{pH}$ conditions was the same.

\section{Bi-directional transport studies of isoflavone solutions in the Caco-2 monolayer model}

The loading concentrations for the three isoflavones solutions in Caco-2 transport studies were $18.25 \pm 0.11$ $\mu \mathrm{g} / \mathrm{ml}$ for daidzein, $22.10 \pm 1.11 \mu \mathrm{g} / \mathrm{ml}$ for puerarin and $18.55 \pm 0.34 \mu \mathrm{g} / \mathrm{ml}$ for daidzin. The results of bi-directional transport studies of the three selected isoflavones are summarized in Table 1. Daidzein was detectable in receiver chambers 15 minutes after being loaded with similar $P_{a p p}$ and percentage recoveries for both directional transport studies. On the other hand, puerarin did not demonstrate passive diffusion either in 120 minutes after apical loading or 60 minutes after basolateral loading in the Caco- 2 monolayer model. The cumulative concentrations of puerarin found at 180 minutes in the receiver sides was $0.043 \pm 0.006 \mu \mathrm{M}$ after apical loading and $0.12 \pm 0.02 \mu \mathrm{M}$ after basal loading with the percentage of recoveries above $89 \%$. In summary, from both directional transport studies, puerarin was barely detectable in the receiver sides (only around $0.3 \%$ of puerarin permeable through the Caco- 2 monolayer). Daidzin showed a significant difference $(\mathrm{t}=24.5, P<0.0001)$ between its two directional transport studies with undetectable daidzin in receiver chamber from all sampling

Table 1 Bi-directional transport of the selected isoflavones in Caco-2 monolayer model

\begin{tabular}{lllll}
\hline Isoflavones & \multicolumn{3}{l}{ Apical to basolateral } & \multicolumn{2}{l}{ Basolateral to apical } \\
\cline { 2 - 5 } & $\boldsymbol{P}_{\text {app }}(\mathbf{c m} / \mathbf{s})$ & Recovery (\%) & $\boldsymbol{P}_{\text {app }}$ (cm/s) & Recovery (\%) \\
\hline Daidzein & $34.2 \pm 7.06 \times 10^{-6}$ & $83.12 \pm 5.74$ & $38.6 \pm 1.81 \times 10^{-6}$ & $83.96 \pm 14.1$ \\
Puerarin & $\mathrm{ND}$ & $88.91 \pm 7.03$ & $\mathrm{ND}$ & $92.73 \pm 8.09$ \\
Daidzin & $\mathrm{ND}$ & $92.94 \pm 1.77$ & $5.30 \pm 0.20 \times 10^{-7}$ & $99.17 \pm 2.55$ \\
\hline
\end{tabular}

The data are expressed as mean \pm SD $(n=3)$. ND: not detectable 
times after apical loading and a more efficient transport after basolateral loading. Therefore, carrier mediated transport of daidzin from $\mathrm{B} \rightarrow \mathrm{A}$ side was suggested and the inhibition transport study on daidzin using MK571 was performed afterwards.

\section{$B \rightarrow A$ transport study of daidzin in the presence of MK571}

Figure 2 shows the cumulative concentration versus time profiles for the basolateral-to-apical transport of daidzin in the absence and presence of MK571. In the presence of MK 571, daidzin was only detectable in the receiver chamber starting from 90 minutes after being loaded. The cumulative concentration of daidzin in the receiver chamber was $0.41 \pm 0.02 \mu \mathrm{M}$ at 180 minutes, which was about $50 \%$ lower than that without MK571. The percentages of recovery of daidzin for these transport studies were all above $90 \%$.

\section{Hydrolysis of daidzin and puerarin during intestinal absorptions}

As shown in Figures 3 and 4, after incubation with rat intestinal microvilli preparations for two hours, the majority of puerarin remained unchanged (Figure 4) whereas daidzin (Figure 3) were completely hydrolyzed to daidzein with no detectable daidzin in the incubation medium.

\section{Discussion}

Daidzein, puerarin, and daidzin, the three selected isoflavones for this study, have a common isoflavone nucleus and structurally exist as aglycone, $\mathrm{C}$-glucoside and $\mathrm{O}$ glucoside respectively. The results of this study show that these three compounds transported differently across the Caco- 2 cell monolayer, thereby exhibiting different transport mechanism when absorbed through the human gastrointestinal tract. The physicochemical tests performed on the three compounds provided information on the properties of the selected isoflavones, which possibly affected their transport through the Caco-2 cell monolayer.

As indicated by the rankings of $D$ at both pH6.8 and pH7.4, there was a large difference (over 200-fold, $P<$ 0.0001 ) in the $D$ between isoflavone aglycone and its glycosides. Daidzein was the most lipid-soluble one with the concentration of daidzein barely detectable in the aqueous layer. Therefore, the $\log P$ and $\log D$ values of daidzein were estimated based on the HPLC detection limit of it. Daidzin demonstrated a moderate $\log P$ value with a slight preference in octanol. Puerarin had the lowest $\log P$ value among the three, suggesting its preference in water.

The bi-directional transport studies of the three selected isoflavones in the Caco-2 monolayer model found that the $P_{a p p}$ values of daidzein were the

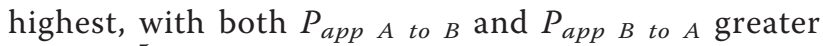
than $10^{-5} \mathrm{~cm} / \mathrm{s}$. Moreover, the $P_{\text {app }}$ value of daidzein found in both directional transport studies were similar, indicating that the compound was likely to be mainly transported across the monolayer by passive diffusion. The percentage of recovery for daidzein after transport studies was about $83 \%$ for both directional transport studies, which was lower than that obtained from puerarin and daidzin. Since the three isoflavones were all confirmed to be stable in $\mathrm{PBS}^{+}$under the experimental condition, the cell uptake and intracellular metabolism of daidzein may contribute to its relatively lower recovery, which was also suggested by Murota et al. [17].

In this study, puerarin was poorly transported through the Caco-2 cell monolayer with its concentration in receiver chamber not being detectable until two hours. Only about $0.3 \%$ of puerarin passed through the monolayer in directional transport studies, indicating that the two directional permeabilities of puerarin were similar and puerarin likely passed through the monolayer with the passive diffusion pathway. Assuming that puerarin was mainly absorbed in the gut by passive diffusion, we think the role of intestinal bacteria in breaking down it into daidzein may be important. However, no hydrolysis of puerarin was shown by Yasuda et al. [12] and this study. The absorption mechanism of puerarin was probably different from that of the other studied isoflavones. This study only established the preliminary absorption profile of puerarin, further investigations are needed to study the relationship between absorbed puerarin and its pharmacological effects.

Daidzin had a different transport profile from the other two isoflavones with a significant efflux medicated by MRP, which was consistent with Vaidyanthan et al [11]. As multidrug resistance-associated protein 2 (MRR2) was responsible to transport various compounds containing $\mathrm{O}$-glucuronides including estradiol $17 \beta$-D-glucuronide [18], it was also possible to mediate the efflux transport of daidzin, due to their structural similarity.

Two factors may contribute to the absorption differences between daidzein and daidzin. Izumi et al. [5] suggested that daidzin is a more hydrophilic and bigger molecular in molecular size which may affect its absorption through passive diffusion pathway. Another factor was the efflux of daidzin, which would prevent it from being transported across the intestinal wall. These two factors may explain the different findings in the absorption of daidzein and daidzin. On the other hand, as active transport mechanisms could not be found in the transport of puerarin, the limited absorption of puerarin was likely due to its large molecular size and high hydrophilicity. Between the absorptions of daidzin and 


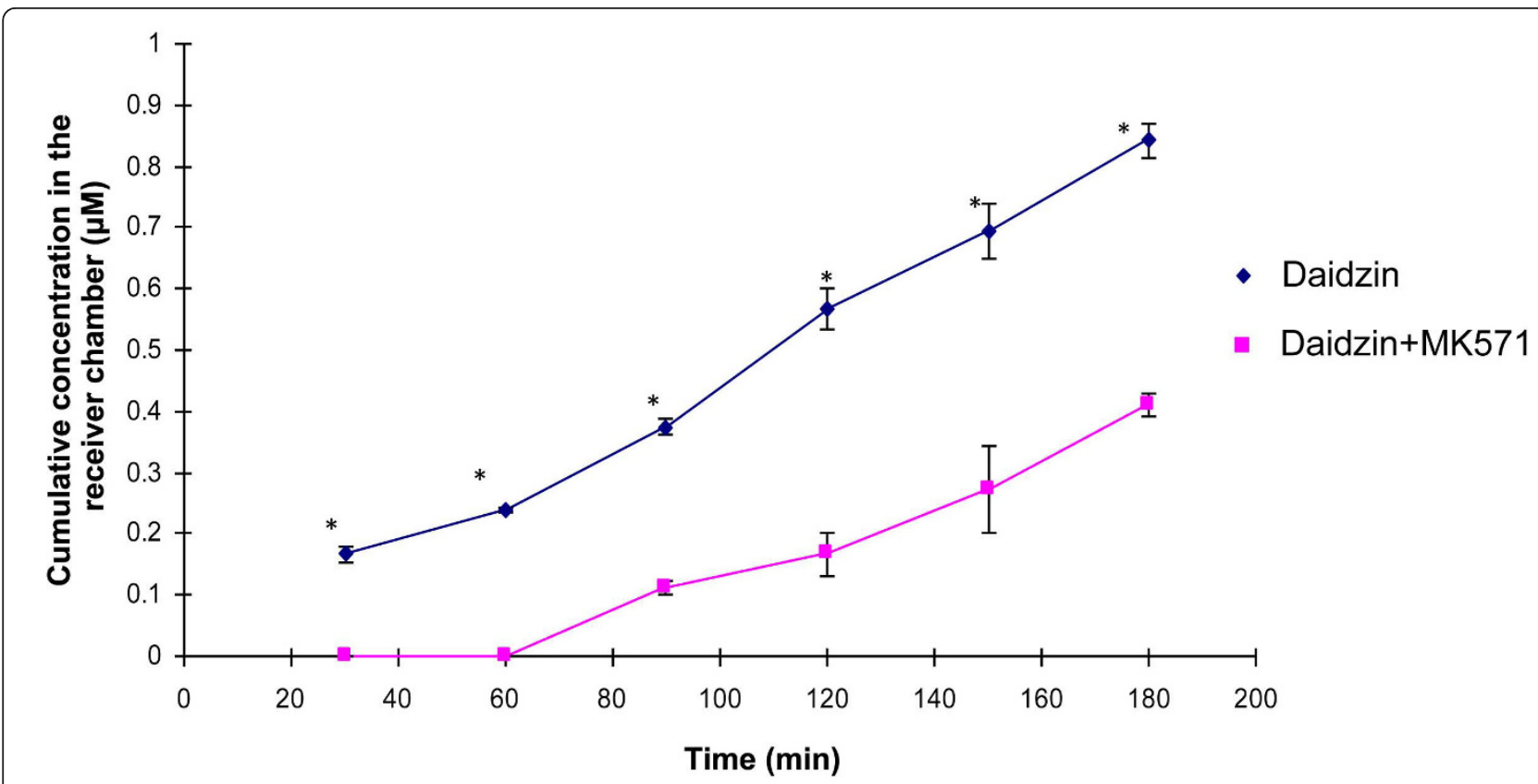

Figure 2 Basolateral-to-apical transport of daidzin in the absence and presence of MK 571, the MRP inhibitors.
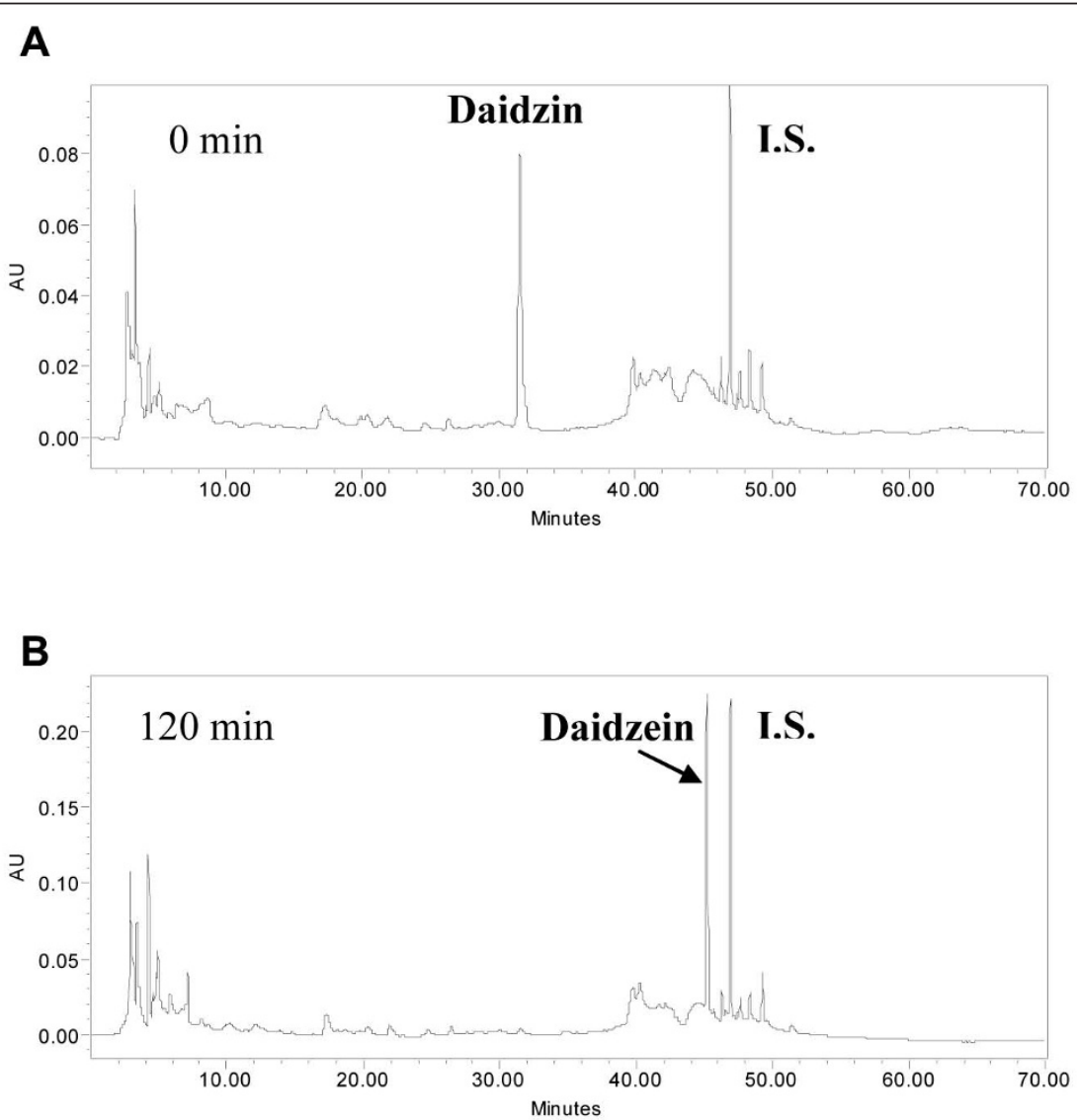

Figure 3 The HPLC-UV chromatograms of daidzin before and after incubations with rat intestinal microvilli preparations. I.S.: internal standard. 

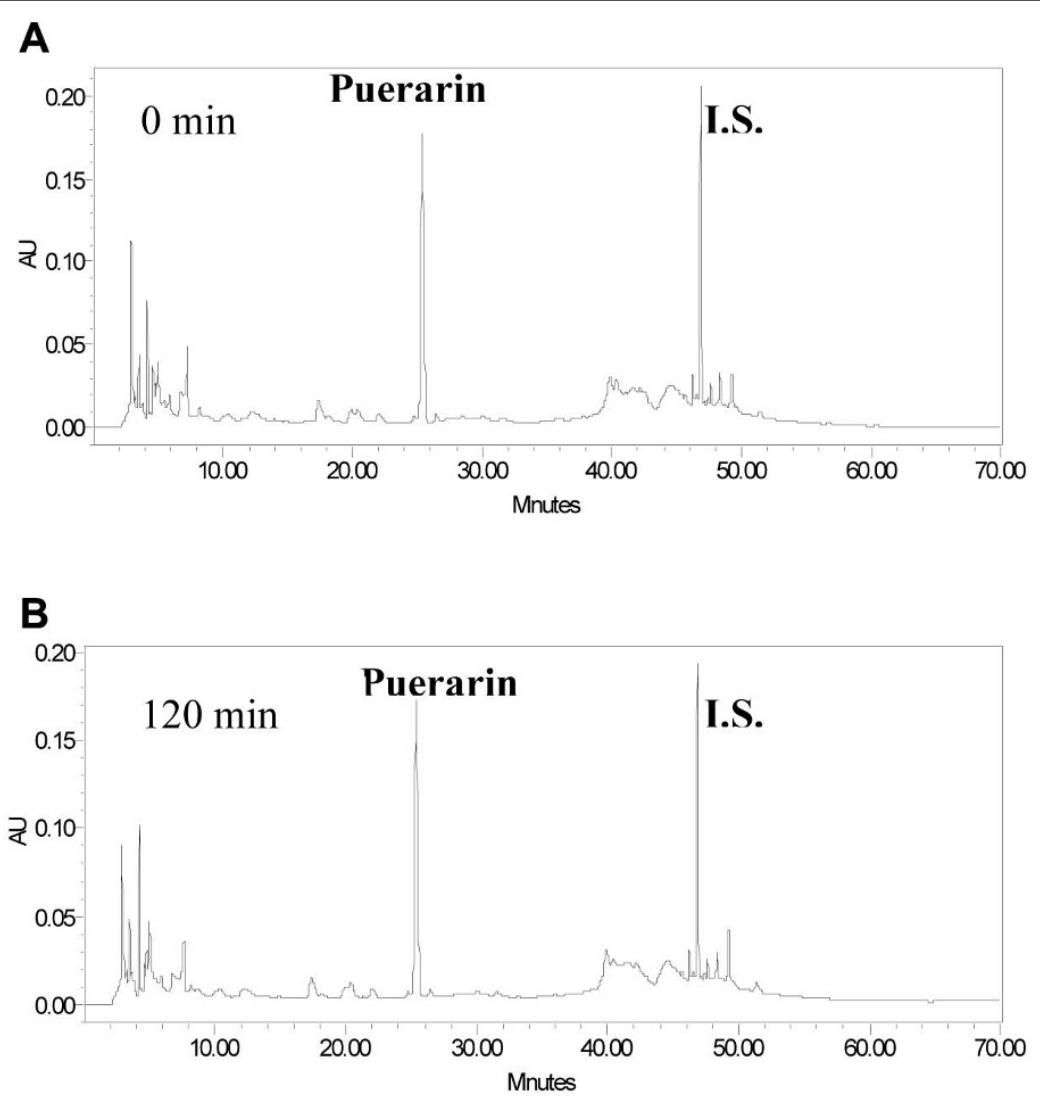

Figure 4 The HPLC-UV chromatograms of puerarin before and after incubations with rat intestinal microvilli preparations. I.S.: internal standard.

puerarin, daidzin showed a significant $\mathrm{B} \rightarrow \mathrm{A}$ efflux whereas puerarin did not, indicating that puerarin was unlikely to be the substrate of MRP transporters.

The incubation with rat intestinal microvilli preparations demonstrated that daidzin could release its aglycone daidzein while puerarin was relatively stable during their absorption processes in. Due to the high polarities and poor $\mathrm{A} \rightarrow \mathrm{B}$ permeabilities of daidzin and puerarin, it was not likely that these two glycosides of daidzein could be absorbed directly at small intestine in vivo. However, daidzin could be absorbed after hydrolysis at small intestine to daidzein which could be absorbed due to its high lipophilicity.

\section{Conclusion}

While daidzein was transported more efficiently, puerarin was resistant to intestinal hydrolysis and inefficiently transported across intestinal epithelium. Daidzin demonstrated a low intestinal absorbability due to a significant efflux transport mediated by MRPs. Daidzin was likely to be hydrolyzed by intestinal microvilli and subsequently released daidzein for intestinal absorption.
Acknowledgements

This study was supported by a CUHK Direct Grant (CUHK 2040830).

\section{Author details}

${ }^{1}$ School of Pharmacy, Faculty of Medicine, The Chinese University of Hong Kong, Shatin, New Territories, Hong Kong SAR, China. ${ }^{2}$ School of Biomedical Sciences, Faculty of Medicine, The Chinese University of Hong Kong, Shatin, New Territories, Hong Kong SAR, China.

\section{Authors' contributions}

$G L$ and $Z Z$ designed the study. LZ and AKPS carried out the experiments. LZ, AKPS and ZZ: performed the data analysis. All authors have read and approved the final manuscript.

\section{Competing interests}

The authors declare that they have no competing interests.

Received: 3 August 2011 Accepted: 23 November 2011 Published: 23 November 2011

\section{References}

1. Keung WM, Klyosov AA, Vallee BL: Daidzin inhibits mitochondrial aldehyde dehydrogenase and suppresses ethanol intake of Syrian golden hamsters. Proc Natl Acad Sci USA 1997, 94:1675-1679.

2. Keung WM, Vallee BL: Daidzin and its antidipsotropic analogs inhibit serotonin and dopamine metabolism in isolated mitochondria. Proc Natl Acad Sci USA 1998, 95:2198-2203.

3. Busby MG, Jeffcoat AR, Bloedon LT, Koch MA, Black T, Dix KJ, Heizer WD, Thomas BF, Hill JM, Crowell JA, Zeisel SH: Clinical characteristics and 
pharmacokinetics of purified soy isoflavones: single-dose administration to healthy men. Am J Clin Nutr 2002, 75:126-136.

4. Lamartiniere CA, Wang J, Smith-Johnson M, Eltoum IE: Daidzein: bioavailability, potential for reproductive toxicity, and breast cancer chemoprevention in female rats. Toxicol Sci 2002, 65:228-238.

5. Izumi T, Piskula MK, Osawa S, Obata A, Tobe K, Saito M, Kataoka S, Kubota Y, Kikuchi M: Soy isoflavone aglycones are absorbed faster and in higher amounts than their glucosides in humans. J Nutr 2000, 130:1695-1699.

6. Setchell KD, Brown NM, Zimmer-Nechemias L, Brashear WT, Wolfe BE, Kirschner AS, Heubi JE: Evidence for lack of absorption of soy isoflavone glycosides in humans, supporting the crucial role of intestinal metabolism for bioavailability. Am J Clin Nutr 2002, 76:447-453.

7. Ohta A, Uehara M, Sakai K, Takasaki M, Adlercreutz H, Morohashi T, Ishimi Y: A combination of dietary fructooligosaccharides and isoflavone conjugates increases femoral bone mineral density and equol production in ovariectomized mice. J Nutr 2002, 132:2048-2054.

8. Watanabe S, Yamaguchi M, Sobue T, Takahashi T, Miura T, Arai Y, Mazur W, Wahala K, Adlercreutz H: Pharmacokinetics of soybean isoflavones in plasma, urine and feces of men after ingestion of $60 \mathrm{~g}$ baked soybean powder (kinako). J Nutr 1998, 128:1710-1715.

9. Richelle M, Pridmore-Merten S, Bodenstab S, Enslen M, Offord EA: Hydrolysis of isoflavone glycosides to aglycones by beta-glycosidase does not alter plasma and urine isoflavone pharmacokinetics in postmenopausal women. J Nutr 2002, 132:2587-2592.

10. Suzuki H, Sugiyama Y: Role of metabolic enzymes and efflux transporters in the absorption of drugs from the small intestine. Eur J Pharm Sci 2000, 12:3-12.

11. Vaidyanathan $\mathrm{JB}$, Walle $\mathrm{T}$ : Transport and metabolism of the tea flavonoid (-)-epicatechin by the human intestinal cell line Caco-2. Pharm Res 2001, 18:1420-1425.

12. Yasuda T, Kano Y, Saito K, Ohsawa K: Urinary and biliary metabolites of puerarin in rats. Biol Pharm Bull 1995, 18:300-303.

13. Kim DH, Yu KU, Bae EA, Han MJ: Metabolism of puerarin and daidzin by human intestinal bacteria and their relation to in vitro cytotoxicity. Biol Pharm Bull 1998, 21:628-630.

14. Artursson P: Epithelial transport of drugs in cell culture. I: A model for studying the passive diffusion of drugs over intestinal absorptive (Caco2) cells. J Pharm Sci 1990, 79:476-482.

15. Zhang L, Zheng Y, Chow MS, Zuo Z: Investigation of intestinal absorption and disposition of green tea catechins by Caco-2 monolayer model. Int $J$ Pharm 2004, 287:1-12.

16. Chang Q, Zuo Z, Chow MS, Ho WK: Difference in absorption of the two structurally similar flavonoid glycosides, hyperoside and isoquercitrin, in rats. Eur J Pharm Biopharm 2005, 59:549-555.

17. Murota K, Shimizu S, Miyamoto S, Izumi T, Obata A, Kikuchi M, Terao J: Unique uptake and transport of isoflavone aglycones by human intestinal caco-2 cells: comparison of isoflavonoids and flavonoids. J Nutr 2002, 132:1956-1961

18. Konig J, Nies AT, Cui Y, Leier I, Keppler D: Conjugate export pumps of the multidrug resistance protein (MRP) family: localization, substrate specificity, and MRP2-mediated drug resistance. Biochim Biophys Acta 1999, 1461:377-394.

doi:10.1186/1749-8546-6-41

Cite this article as: Zhang et al: Intestinal absorbability of three Radix Puerariae isoflavones including daidzein, daidzin and puerarin. Chinese Medicine 2011 6:41.

\section{Submit your next manuscript to BioMed Central and take full advantage of:}

- Convenient online submission

- Thorough peer review

- No space constraints or color figure charges

- Immediate publication on acceptance

- Inclusion in PubMed, CAS, Scopus and Google Scholar

- Research which is freely available for redistribution 